\title{
Deep sea tests of a prototype of the KM3NeT digital optical module
}

\section{KM3NeT Collaboration}

S. Adrián-Martínez ${ }^{1}$, M. Ageron², F. Aharonian ${ }^{3}$, S. Aiello ${ }^{4}$, A. Albert ${ }^{5}$, F. Ameli ${ }^{6}$, E. G. Anassontzis ${ }^{7}$, M. Anghinolfi ${ }^{8}$, G. Anton ${ }^{9}$, S. Anvar ${ }^{10}$, M. Ardid ${ }^{1}$, R. de Asmundis ${ }^{11}$, K. Balasi ${ }^{12}$, H. Band ${ }^{13}$, G. Barbarino ${ }^{11,14}$, E. Barbarito ${ }^{15}$, F. Barbato ${ }^{11,14}$, B. Baret ${ }^{16}$, S. Baron ${ }^{16}$, A. Belias ${ }^{12,17}$, E. Berbee ${ }^{13}$, A. M. van den Berg ${ }^{18}$, A. Berkien ${ }^{13}$, V. Bertin ${ }^{2}$, S. Beurthey ${ }^{2}$, V. van Beveren ${ }^{13}$, N. Beverini ${ }^{19,20}$, S. Biagi $^{21,22}$, S. Bianucci ${ }^{20}$, M. Billault ${ }^{2}$, A. Birbas ${ }^{23}$, H. Boer Rookhuizen ${ }^{13}$, R. Bormuth ${ }^{13,24}$, V. Bouché ${ }^{6,25}$, B. Bouhadef ${ }^{20}$, G. Bourlis ${ }^{23}$, M. Bouwhuis ${ }^{13}$, C. Bozza ${ }^{14,26}$, R. Bruijn ${ }^{13,27}$, J. Brunner ${ }^{2}$, G. Cacopardo ${ }^{28}$, L. Caillat ${ }^{2}$, M. Calamai ${ }^{20}$, D. Calvo ${ }^{30}$, A. Capone ${ }^{25}$, L. Caramete ${ }^{31}$, F. Caruso ${ }^{28}$, S. Cecchini ${ }^{21,22}$, A. Ceres $^{15}$, R. Cereseto ${ }^{8}$, C. Championn ${ }^{16}$, F. Château ${ }^{10}$, T. Chiarusi ${ }^{21}$, B. Christopoulou ${ }^{23}$, M. Circella ${ }^{15}$, L. Classen ${ }^{9}$, R. Cocimano ${ }^{28}$, S. Colonges $^{16}$, R. Coniglione $^{28}$, A. Cosquer ${ }^{2}$,

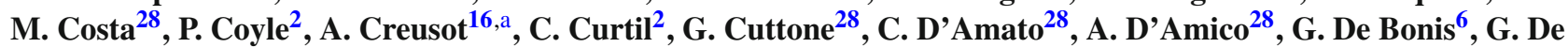
Rosa $^{11,14}$, N. Deniskina ${ }^{11}$, J.-J. Destelle ${ }^{2}$, C. Distefano ${ }^{28}$, C. Donzaud ${ }^{16,29}$, D. Dornic ${ }^{2}$, Q. Dorosti-Hasankiadeh ${ }^{18}$, E. Drakopoulou ${ }^{12}$, D. Drouhin ${ }^{5}$, L. Drury ${ }^{3}$, D. Durand ${ }^{10}$, T. Eberl ${ }^{9}$, C. Eleftheriadis ${ }^{32}$, D. Elsaesser ${ }^{33}$,

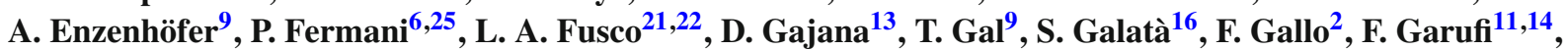
M. Gebyehu ${ }^{13}$, V. Giordano ${ }^{4}$, N. Gizani' ${ }^{23}$, R. Gracia Ruiz' ${ }^{16}$, K. Graf ${ }^{9}$, R. Grasso ${ }^{28}$, G. Grella ${ }^{14,26}$, A. Grmek ${ }^{28}$, R. Habel ${ }^{34}$, H. van Haren ${ }^{35}$, T. Heid ${ }^{9}$, A. Heijboer ${ }^{13}$, E. Heine ${ }^{13}$, S. Henry ${ }^{2}$, J. J. Hernández-Rey ${ }^{30}$, B. Herold ${ }^{9}$, M. A. Hevinga ${ }^{18}$, M. van der Hoek ${ }^{13}$, J. Hofestädt ${ }^{9}$, J. Hogenbirk ${ }^{13}$, C. Hugon ${ }^{8}$, J. Hößl ${ }^{9}$, M. Imbesi ${ }^{28}$, C. James ${ }^{9}$, P. Jansweijer ${ }^{13}$, J. Jochum ${ }^{36}$, M. de Jong ${ }^{13,24}$, M. Kadler ${ }^{33}$, O. Kalekin ${ }^{9}$, A. Kappes ${ }^{9}$, E. Kappos ${ }^{7,12}$, U. Katz ${ }^{9}$, O. Kavatsyuk ${ }^{18}$, P. Keller ${ }^{2}$, G. Kieft ${ }^{13}$, E. Koffeman ${ }^{13,27}$, H. Kok ${ }^{13}$, P. Kooijman ${ }^{13,27,37, b}$, J. Koopstra ${ }^{13}$,

A. Korporaal ${ }^{13}$, A. Kouchner ${ }^{16}$, S. Koutsoukos ${ }^{17}$, I. Kreykenbohm ${ }^{38}$, V. Kulikovskiy ${ }^{8}$, R. Lahmann' ${ }^{9}$, P. Lamare ${ }^{2}$, G. Larosa $^{28}$, D. Lattuada ${ }^{28}$, H. Le Provost ${ }^{10}$, A. Leisos ${ }^{23}$, D. Lenis ${ }^{23}$, E. Leonora ${ }^{4}$, M. Lindsey Clark ${ }^{16}$, A. Liolios ${ }^{32}$, C. D. Llorens Alvarez ${ }^{1}$, H. Löhner ${ }^{18}$, D. Lo Presti ${ }^{4,39}$, F. Louis ${ }^{10}$, E. Maccioni ${ }^{19}$, K. Mannheim ${ }^{33}$, K. Manolopoulos ${ }^{7,12}$, A. Margiotta ${ }^{21,22}$, O. Mariş ${ }^{31}$, C. Markou ${ }^{12}$, J. A. Martínez-Mora ${ }^{30}$, A. Martini ${ }^{34}$, R. Masullo6,25 ${ }^{6}$ T. Michael ${ }^{13}$, P. Migliozzi ${ }^{11}$, E. Migneco ${ }^{28}$, A. Miraglia ${ }^{28}$, C. Mollo $^{11}$, M. Mongelli ${ }^{15}$, M. Morganti ${ }^{20, c}$,

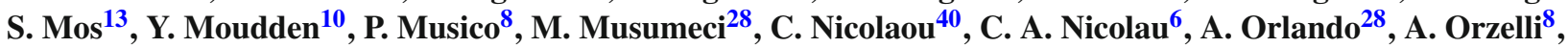
K. Papageorgiou ${ }^{41}$, A. Papaikonomou ${ }^{12,23}$, R. Papaleo ${ }^{28}$, G. E. Păvălaşs ${ }^{31}$ H. Peek ${ }^{13}$, C. Pellegrino ${ }^{28}$, M. G. Pellegriti ${ }^{28}$, C. Perrina ${ }^{6,25}$, C. Petridou ${ }^{32}$, P. Piattelli ${ }^{28}$, K. Pikounis ${ }^{12}$, V. Popa ${ }^{31}$, Th. Pradier ${ }^{42}$, M. Priede ${ }^{43}$, G. Pühlhofer ${ }^{36}$, S. Pulvirenti ${ }^{28}$, C. Racca $^{5}$, F. Raffaelli ${ }^{20}$, N. Randazzo ${ }^{4}$, P. A. Rapidis ${ }^{12,17}$, P. Razis ${ }^{40}$, D. Real ${ }^{30}$, L. Resvanis ${ }^{7,17}$, J. Reubelt ${ }^{9}$, G. Riccobene ${ }^{28}$, A. Rovelli28, J. Royon ${ }^{2}$, M. Saldaña ${ }^{1}$, D. F. E. Samtleben ${ }^{13,24}$, M. Sanguineti ${ }^{44}$, A. Santangelo ${ }^{36}$, P. Sapienza ${ }^{28}$, I. Savvidis ${ }^{32}$, J. Schmelling ${ }^{13}$, J. Schnabel ${ }^{9}$, M. Sedita ${ }^{28}$, T. Seitz ${ }^{9}$, I. Sgura ${ }^{15}$, F. Simeone ${ }^{6}$, I. Siotis ${ }^{12}$, V. Sipala ${ }^{4}$, M. Solazzo ${ }^{2}$, A. Spitaleri28, M. Spurio ${ }^{21,22}$, G. Stavropoulos ${ }^{12}$, J. Steijger ${ }^{13}$, T. Stolarczyk ${ }^{45}$, D. Stransky ${ }^{9}$, M. Taiuti ${ }^{8,44}$, G. Terreni ${ }^{20}$, D. Tézier ${ }^{2}$, S. Théraube $^{2}$, L. F. Thompson ${ }^{46}$,

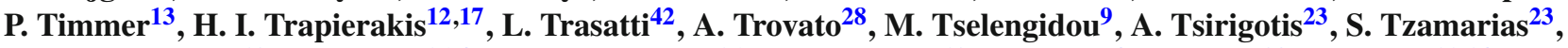
E. Tzamariudaki ${ }^{12}$, B. Vallage ${ }^{16,45}$, V. Van Elewyck ${ }^{16}$, J. Vermeulen ${ }^{13}$, P. Vernin ${ }^{45}$, S. Viola ${ }^{28}$, D. Vivolo ${ }^{11,14}$, P. Werneke $^{13}$, L. Wiggers ${ }^{13}$, J. Wilms ${ }^{38}$, E. de Wolf ${ }^{13,27}$, R. H. L. van Wooning ${ }^{18}$, K. Yatkin ${ }^{2}$, K. Zachariadou ${ }^{47}$, E. Zonca ${ }^{10}$, J. D. Zornoza ${ }^{30}$, J. Zúñiga ${ }^{30}$, A. Zwart ${ }^{13}$

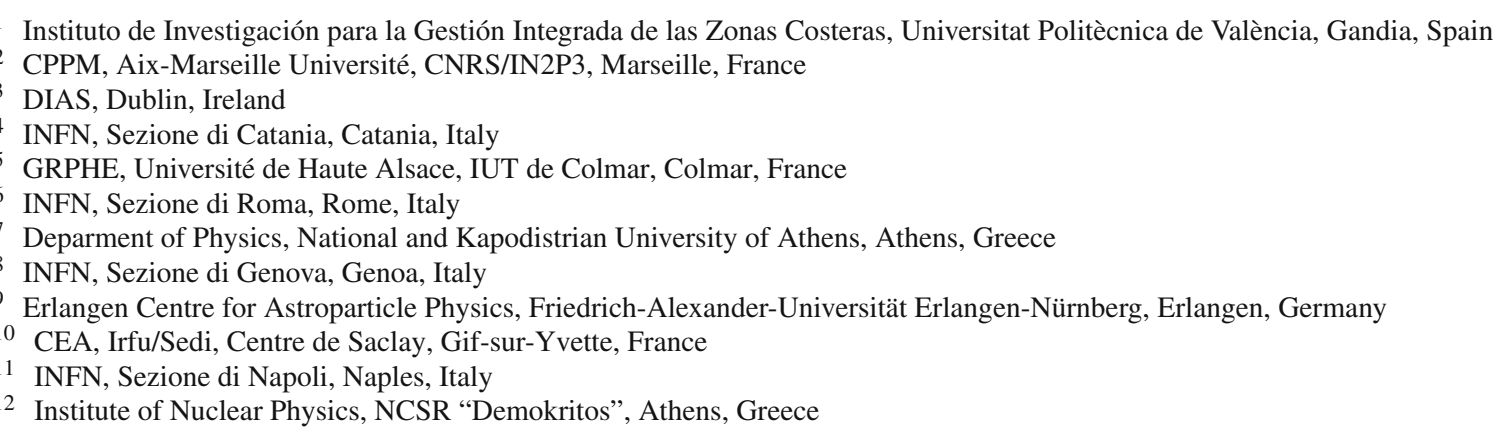


13 Nikhef, Amsterdam, The Netherlands

14 Dipartimento di Fisica, Università 'Federico II', Naples, Italy

15 INFN, Sezione di Bari, Bari, Italy

16 APC,Université Paris Diderot, CNRS/IN2P3, CEA/IRFU Observatoire de Paris, Sorbonne Paris Cité, 75205, Paris, France

17 NESTOR Institute for Deep Sea Research, Technology, and Neutrino Astroparticle Physics, National Observatory of Athens, Pylos, Greece

18 KVI-CART, University of Groningen, Groningen, The Netherlands

19 INFN, Sezione di Pisa, Pisa, Italy

20 Dipertimento di Fisica, Università di Pisa, Pisa, Italy

21 INFN, Sezione di Bologna, Bologna, Italy

22 Dipartimento di Fisica e Astronomia, Universitá di Bologna, Bologna, Italy

23 School of Science and Technology, Hellenic Open University, Patras, Greece

24 Leiden Institute of Physics, Leiden University, Leiden, The Netherlands

5 Dipartimento di Fisica, Università di Roma La Sapienza, Rome, Italy

Dipartimento di Fisica, Università di Salerno, Fisciano, Italy

7 Institute of Physics, University of Amsterdam, Amsterdam, The Netherlands

28 INFN, Laboratori Nazionali del Sud, Catania, Italy

9 Université Paris-Sud, 91405, Orsay Cedex, France

IFIC-Instituto de Física Corpuscular, (CSIC-Universitat de València), Valencia, Spain

Institute of Space Science, Bucharest, Romania

Aristotle University Thessaloniki, Thessaloniki, Greece

University Würzburg, Würzburg, Germany

INFN, Laboratori Nazionali di Frascati, Frascati, Italy

NIOZ, Texel, The Netherlands

36 Eberhard Karls Universität Tübingen, Tübingen, Germany

7 Utrecht University, Utrecht, The Netherlands

8 Dr. Remeis Sternwarte, Friedrich-Alexander-Universität Erlangen-Nürnberg, Bamberg, Germany

Dipartimento di Fisica ed Astronomia, Università di Catania, Catania, Italy

Physics Department, University of Cyprus, Nicosia, Cyprus

1 University of Aegean, Athens, Greece

IPHC, CNRS/IN2P3, Strasbourg, France

Oceanlab, University of Aberdeen, Aberdeen, UK

Dipartimento di Fisica, Università di Genova, Genoa, Italy

5 CEA, Irfu/SPP, Centre de Saclay, Gif-sur-Yvette, France

46 Department of Physics and Astronomy, University of Sheffield, Sheffield, UK

47 Technological Education Institute of Pireaus, Piraeus, Greece

Received: 26 May 2014 / Accepted: 22 August 2014 / Published online: 26 September 2014

(c) The Author(s) 2014. This article is published with open access at Springerlink.com

\begin{abstract}
The first prototype of a photo-detection unit of the future KM3NeT neutrino telescope has been deployed in the deep waters of the Mediterranean Sea. This digital optical module has a novel design with a very large photocathode area segmented by the use of 31 three inch photomultiplier tubes. It has been integrated in the ANTARES detector for in-situ testing and validation. This paper reports on the first months of data taking and rate measurements. The analysis results highlight the capabilities of the new module design in terms of background suppression and signal recognition. The directionality of the optical module enables the recognition of multiple Cherenkov photons from the same ${ }^{40} \mathrm{~K}$ decay and the localisation of bioluminescent activity in the neighbourhood. The single unit can cleanly identify atmospheric muons and provide sensitivity to the muon arrival directions.
\end{abstract}

\footnotetext{
a e-mail: creusot@in2p3.fr

b e-mail: h84@nikhef.nl

${ }^{c}$ Also at Accademia Novale di Livorno, Livorno, Italy
}

\section{Introduction}

The KM3NeT Observatory [1] is a large scale neutrino telescope to be built in the deep waters of the Mediterranean Sea. With four to five cubic kilometres instrumented with thousands of optical sensors, KM3NeT will be the largest and most sensitive neutrino detector in the energy range of 1-10000 TeV. It will be capable of neutrino astronomy with unprecedented accuracy. Being situated in the Northern Hemisphere it will be particularly suited to the investigation of high energy neutrinos from our Galaxy. The IceCube collaboration has recently reported a first signal of neutrinos with energies of PeVs [2]. A subsequent analysis showed indeed an excess of several tens of events that are attributed to extraterrestrial sources [3]. This signal with energies up to $2 \mathrm{PeV}$ strengthens the motivation for the construction of KM3NeT. Several types of astrophysical objects have been proposed as sites where hadrons are accelerated to extreme energies. The interaction of these particles with matter or radiation in or near the source produces pions 
and subsequently high energy neutrinos [4-6]. These neutrinos propagate with almost no interaction and may reach the Earth undisturbed. From the observed neutrino direction the sources may be identified $[7,8]$.

Detection of neutrinos is based on sampling the Cherenkov light induced by the particles produced in a neutrino interaction in the vicinity of the detector. The muon produced in the charged current interaction of a muon-neutrino provides through its long range in water a particularly sensitive detection channel, but the huge volume of KM3NeT also provides large sensitivity to the other neutrino flavours and to the neutral current interactions. The properties of the deep Mediterranean water allow for an accuracy in the determination of the neutrino direction of $\sigma(\Omega) \approx 0.7^{\circ} / \sqrt{E[\mathrm{TeV}]}$ for muon neutrinos and $\sigma(\Omega) \approx 1.1^{\circ}$ for cascade events of $\mathrm{E}>10 \mathrm{TeV}$. The effect of the water properties has been demonstrated in the KM3NeT predecessor ANTARES [9].

The sampling of Cherenkov light in the KM3NeT telescope is performed with the Digital Optical Modules (DOMs). A prototype of these is the subject of this paper. It has been installed on the instrumentation line of the ANTARES detector [10]. A technical drawing of the DOM and a picture of the DOM connected to the ANTARES line during deployment are shown in Fig. 1.

\section{Digital optical module design and deployment}

The DOM consists of a glass sphere, ${ }^{1} 432 \mathrm{~mm}$ in diameter. The sphere houses 31 photomultiplier tubes (PMTs) with a photocathode diameter of $72 \mathrm{~mm}$ [1]. Each tube is surrounded by a cone-shaped reflector that effectively increases the diameter to about $95 \mathrm{~mm}$ [11]. For the present prototype module, ETEL ${ }^{2}$ D783FL PMTs are used. They are arranged in 5 rings of PMTs with zenith angles of $56^{\circ}, 72^{\circ}, 107^{\circ}, 123^{\circ}$ and $148^{\circ}$, respectively (see Fig. 1a). In each ring the 6 PMTs are spaced at $60^{\circ}$ in azimuth and successive rings are staggered by $30^{\circ}$. The last PMT points vertically downward at a zenith angle of $180^{\circ}$.

Each PMT has its own individual very-low-power highvoltage base [12] with integrated amplification and adjustable discrimination. A multi-purpose multi-channel TDC incorporated within an FPGA was developed during the design phase of KM3NeT [13]. A modified version, implemented in the DOM, digitises the arrival time and the width of the discriminated PMT pulse, the time-over-threshold (ToT) [14]. For the results presented in this paper, the threshold is set at the level of 0.3 of the mean single photon pulse height

\footnotetext{
1 Vitrovex, NAUTILUS Marine Service GmbH, Buxtehude, Germany; http://www.nautilus-gmbh.com/vitrovex-deep-sea-housings.

${ }^{2}$ ET Enterprises Ltd, Uxbridge, UB8 2YF, UK. http://www.et-enter prises.com.
}
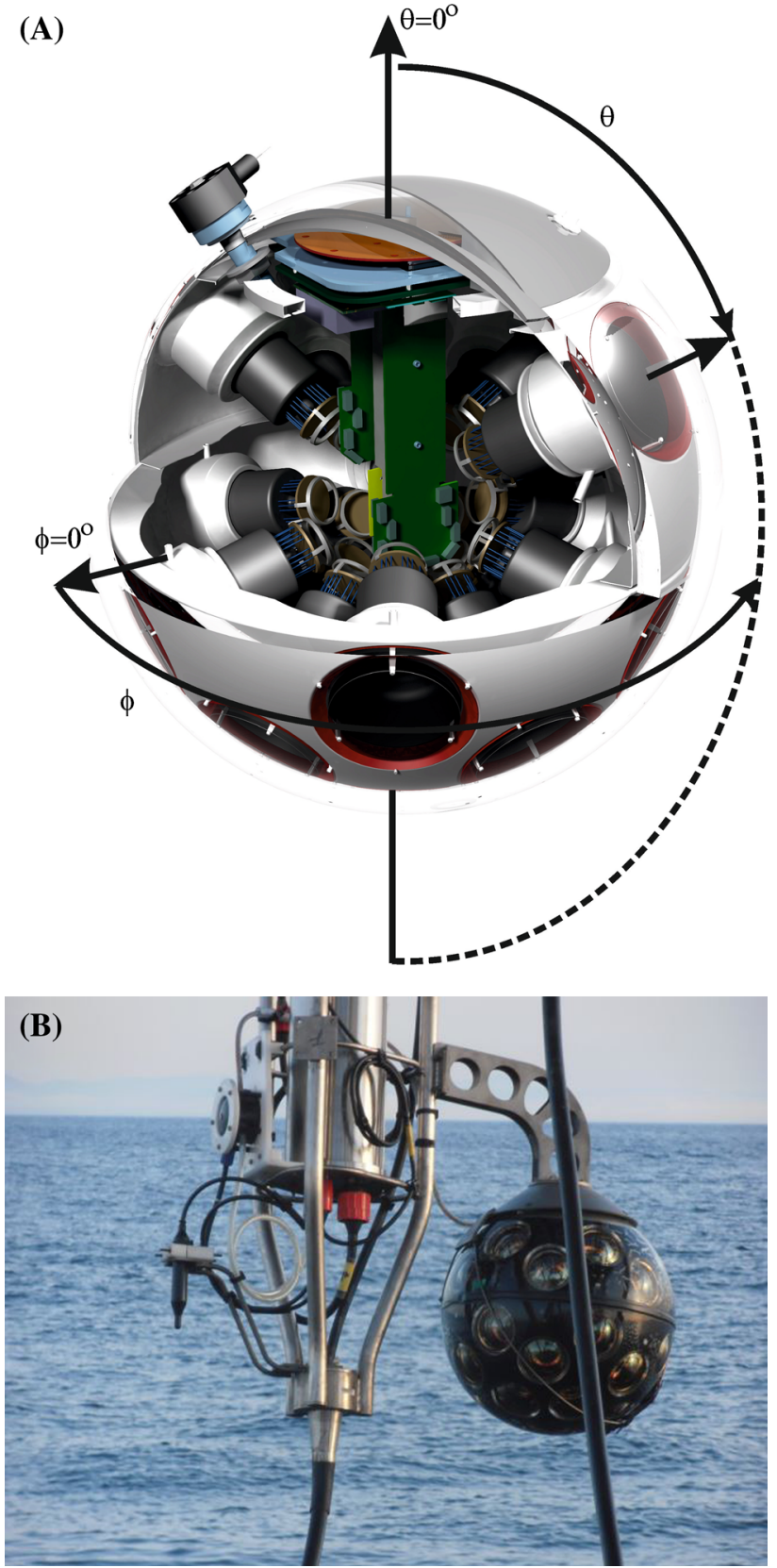

Fig. 1 a A 3D cutout drawing of the DOM. The zenith angle $\theta$ and the azimuthal angle $\phi$ are indicated. Vertically upward corresponds to $\theta=0^{\circ}$ whereas $\phi=0^{\circ}$ points to the support cable. $\mathbf{b}$ The DOM connected to the ANTARES instrumentation line. The structure to which the DOM is connected is a standard ANTARES support frame including a cylindrical electronics container

and the high voltage is set to provide an amplification of $3 \times 10^{6}$. The data are transported the $45 \mathrm{~km}$ to shore through the ANTARES $1 \mathrm{~Gb} / \mathrm{s}$ multiplexed optical link, that uses reflective modulation in the DOM $[15,16]$.

As compared to traditional optical modules with single large PMTs [17-19], the design of the DOM has the advantage that it houses approximately three times the effective 
photocathode area in a single glass sphere and has an almost uniform angular coverage. Because the photocathode is segmented, the arrival of more than one photon at the DOM is identified with high efficiency and purity and provides a sensitivity to the direction of the detected light. This allows for a highly efficient rejection of optical background. The number of pressure resistant vessels is minimised. Because of the low anode current the PMTs are expected to experience little ageing. In addition failure of a single PMT leaves the DOM still $97 \%$ efficient.

The present DOM is the first KM3NeT optical module deployed and operated in the deep sea. For this prototype step, the module was deployed as a complete stand-alone detector on the instrumentation line of ANTARES. This line provides the DOM with power and the optical connection to shore. On the 16th of April 2013 the line with the DOM attached was deployed and connected using a ROV (Remotely Operated submersible Vehicle). The line is situated on the ANTARES detector site, south of Toulon, France $\left(42^{\circ} 50^{\prime} \mathrm{N}, 6^{\circ} 10^{\prime} \mathrm{E}\right)$ at a depth of $2475 \mathrm{~m}$. Being connected to the line approximately $100 \mathrm{~m}$ above the anchor, the DOM operates at a depth of $2375 \mathrm{~m}$. Data taking commenced the day of deployment.

\section{Calibration}

The first data-taking runs were used to perform calibrations of the system and determine the counting rates of the individual PMTs. Figure 2a shows the result of one such calibration run. The graph shows the distribution of the registered ToT for one of the PMTs in the DOM. Two distinct peaks can be identified: one with a ToT attributable to single photon signals at $35 \mathrm{~ns}$, with a spread of $5.5 \mathrm{~ns}$ FWHM, and one with significantly longer ToT caused by the flashing of the ANTARES calibration laser [20] and is equivalent to 8-10 photo-electrons. The small peak at about $15 \mathrm{~ns}$ is most likely an instrumental effect of the pulseshaping circuitry. Figure $2 b$ gives the distribution of the time difference between successive hits, $\Delta T$, on a typical single PMT, showing the expected exponential shape. It has a slope $\tau=1.05 \times 10^{-4} \mathrm{~s}$ corresponding to a purely random background singles rate of around $9.5 \mathrm{kHz}$, mostly due to ${ }^{40} \mathrm{~K}$ (see below). Because of its low repetition frequency of $1 \mathrm{kHz}$ the signals from the laser do not appear in this plot. The increase in the rate near $\Delta T=0$ is due to the approximately $5 \%$ probability that these PMTs produce an afterpulse at around $3 \mu$ s after the initial pulse.

The aggregate hit rates of all PMTs in the DOM are shown in Fig. 3a as a function of the time in $134 \mathrm{~ms}$ timeframe bins, for a run without laser. The baseline corresponds to an average rate of about $8 \mathrm{kHz}$ per PMT and is stable. Some timeframe bins show significant increases in the count rate. These increases can be attributed to bioluminescent activity.
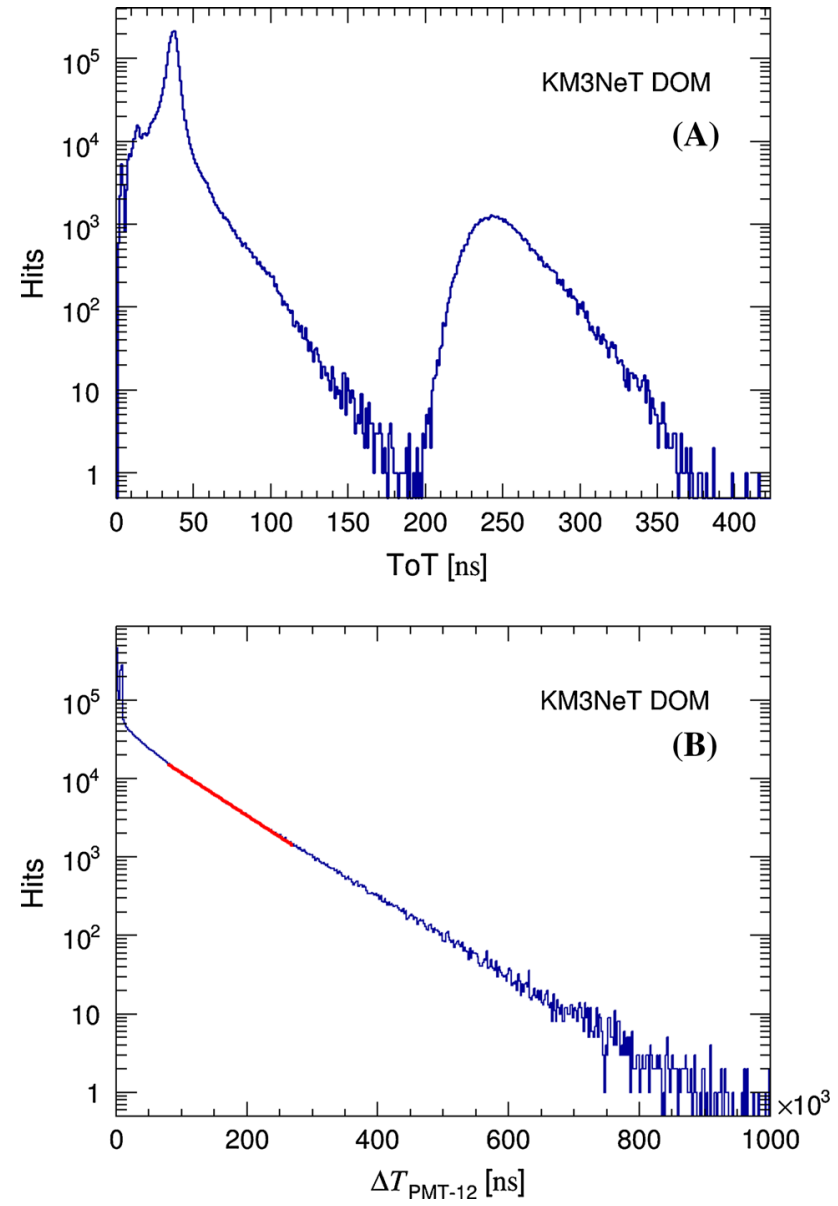

Fig. 2 a Numbers of hits as a function of ToT for one of the PMTs. The broad ToT peak corresponds to the large pulse height caused by the laser calibration. b Histogram of the time difference between two consecutive hits. The exponential fit, as indicated by the red line, gives a characteristic time $\tau=1.05 \times 10^{-4} \mathrm{~s}$

Because the DOM contains many PMTs it is possible to look for coincidences of hits within the single optical module. Figure $3 \mathrm{a}$ also shows the rate for twofold and threefold coincidences as a function of time. A two- (three-) fold coincidence is defined by the occurrence of a hit in two (three) PMTs within a time window of $20 \mathrm{~ns}$. To provide adequate statistics, the rate of threefold coincidences is averaged over three bins. As expected the twofold coincidences exhibit increased rates in the same bins due to the enhanced random coincidence rate. In the threefold coincidences, these increases are significantly diminished. The peaks in the singles rates consist mainly of rate increases of a few PMTs at a time. The activity is not uniformly distributed over all PMTs but shows a preferred direction. A bioluminescent burst of a PMT is defined as a frame that exceeds the high-rate veto cut defined in Fig. 3b. If the preceding frame also fails the cut this is considered as the continuation of a single burst. Figure $3 \mathrm{c}$ shows the occurrence rate of bioluminescent bursts as a function of the PMT position in zenith and azimuth, indicating that 

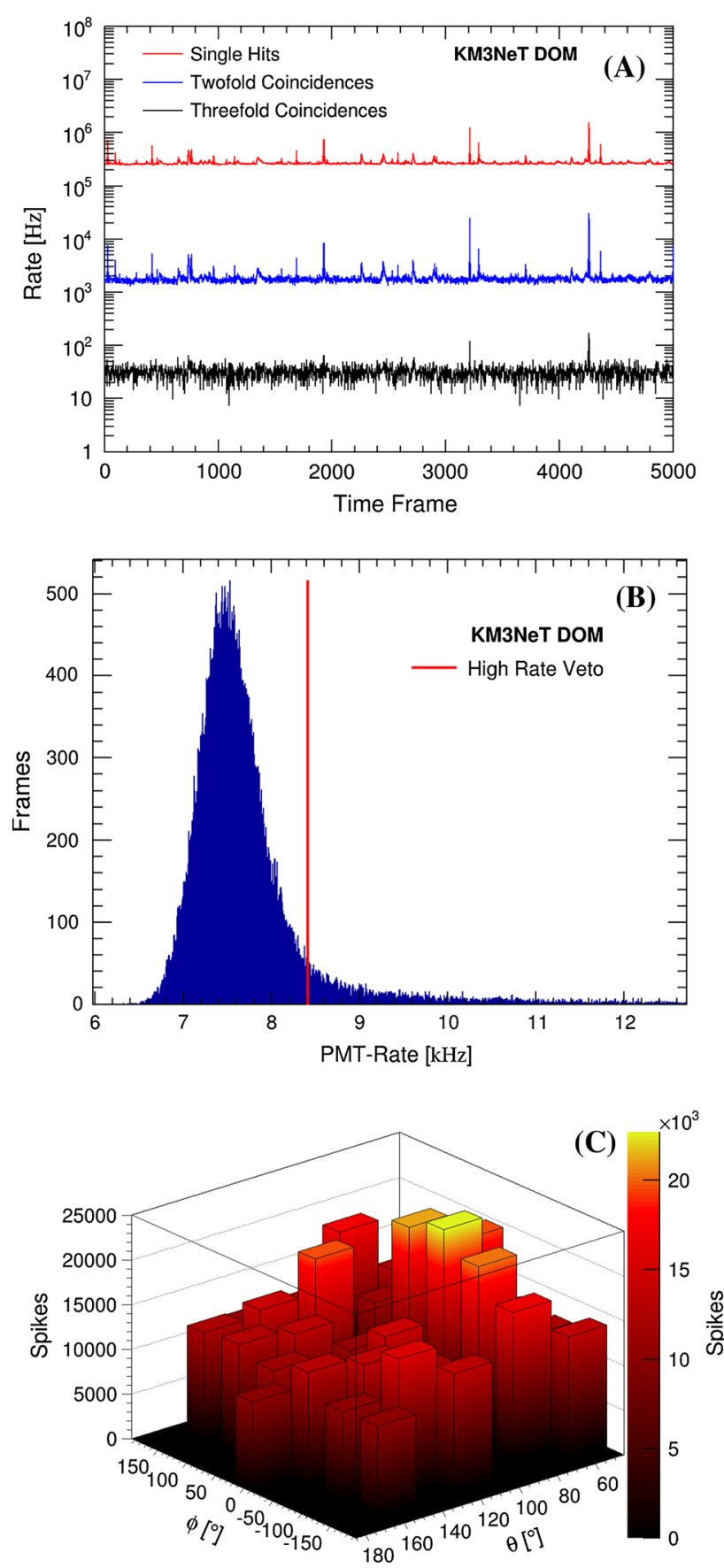

Fig. 3 a Aggregate hit rates as a function of the time measured in $\sim 134$ ms bins. The top trace is for single hits, while the lower traces are for two- and threefold coincidences within a $20 \mathrm{~ns}$ window. b Histogram of the rate measured in $134 \mathrm{~ms}$ timeframe bins for a representative PMT. The rising edge of the histogram is fit to a Gaussian and the cutoff used in the further analysis is set at the mean plus 3 times the standard deviation (indicated by the red line). The cut-off is determined for each PMT separately. $\mathbf{c}$ The rate of bioluminescent bursts as a function of azimuth and zenith angles. The support structure is viewed by PMTs at small $\theta$ and near $\phi=0^{\circ}$

a significant amount of the bioluminescent activity is emanating from the direction of the support structure and electronics container. This type of structure will be absent in the final KM3NeT string, where the DOMs are supported by two thin, $4 \mathrm{~mm}$ diameter ropes. Averaged over the period July to December the rate of bursts per PMT is around one every $20 \mathrm{~s}$ for most PMTs and for the ones facing the structure it is 2.5 times larger. To select a very clean sample of hits, in the following a somewhat rigorous approach has been adopted (high-rate veto). If in any bin a single PMT shows a high rate activity, as defined in Fig. 3b, the bin is removed from the analysis. Depending on the conditions this cut removes on average $10-15 \%$ of the frames.

Seawater contains potassium at the level of $416 \mathrm{ppm}$ (at the ANTARES site) of which $0.0118 \%$ is the radioactive isotope ${ }^{40} \mathrm{~K}\left(\tau_{1 / 2}=1.28 \times 10^{9}\right.$ years $)$. The decay of ${ }^{40} \mathrm{~K}$ yields either $\beta$-electrons or $\gamma$-rays with energies of approximately $1 \mathrm{MeV}$. Electrons, either directly produced or from Compton scattering, induce Cherenkov radiation while also undergoing multiple Coulomb scattering in the surrounding water. These processes altogether constitute an isotropic source of about 100 detectable photons, as determined from simulation. These are the main cause of the baseline singles rate in the PMTs. Assuming a random singles distribution this would yield a baseline twofold coincidence rate of $1200 \mathrm{~Hz}$ whereas the measured rate is around $1600 \mathrm{~Hz}$. The reason is that the DOM is also capable of detecting multiple photons from a single ${ }^{40} \mathrm{~K}$ decay. Figure $4 \mathrm{a}-\mathrm{d}$ show the rates as a function of time difference for increasing angular separation of the PMTs. The clear Gaussian peak centered at a time difference of zero indicates the detection of two photons from the same ${ }^{40} \mathrm{~K}$ decay. The width of the peak is $6 \mathrm{~ns}$ (FWHM) corresponding to a single PMT time resolution of $\sigma=1.8 \mathrm{~ns}$. This signal provides an excellent intra-DOM timing calibration. The peak becomes less prominent as the angular separation increases from $33^{\circ}$ in Fig. $4 \mathrm{a}$ to $65^{\circ}$ in Fig. $4 \mathrm{~b}$ to $120^{\circ}$ in Fig. 4c. When the PMTs are positioned back to back as in Fig. 4d the peak has virtually disappeared leaving only random coincidences. Figure 5 shows the background subtracted coincidence rate as a function of the angular separation between the PMTs, together with the results of a simulation of the ${ }^{40} \mathrm{~K}$ decay [21]. In general there is good agreement between data and simulation, although the rate at small angular separation is overestimated by the simulation at the $10 \%$ level whereas in the data some random coincidences appear at large separation. The average total rates observed in the DOM as a function of run number are given in Fig. 6, without the high rate veto. Each run lasts approximately $10 \mathrm{~min}$ and the figure spans a period of 6 months starting in July 2013. The singles rate in Fig. 6a shows large variations due to the varying bioluminescence component present in the run with an observed maximum rate of $1.2 \mathrm{MHz}$ for the full DOM. As the coincidence level increases, the fluctuations decrease. In general, the rates are observed to decrease with increasing run number corresponding to a decrease in bioluminescent activity from July through December. Through shifting of each 

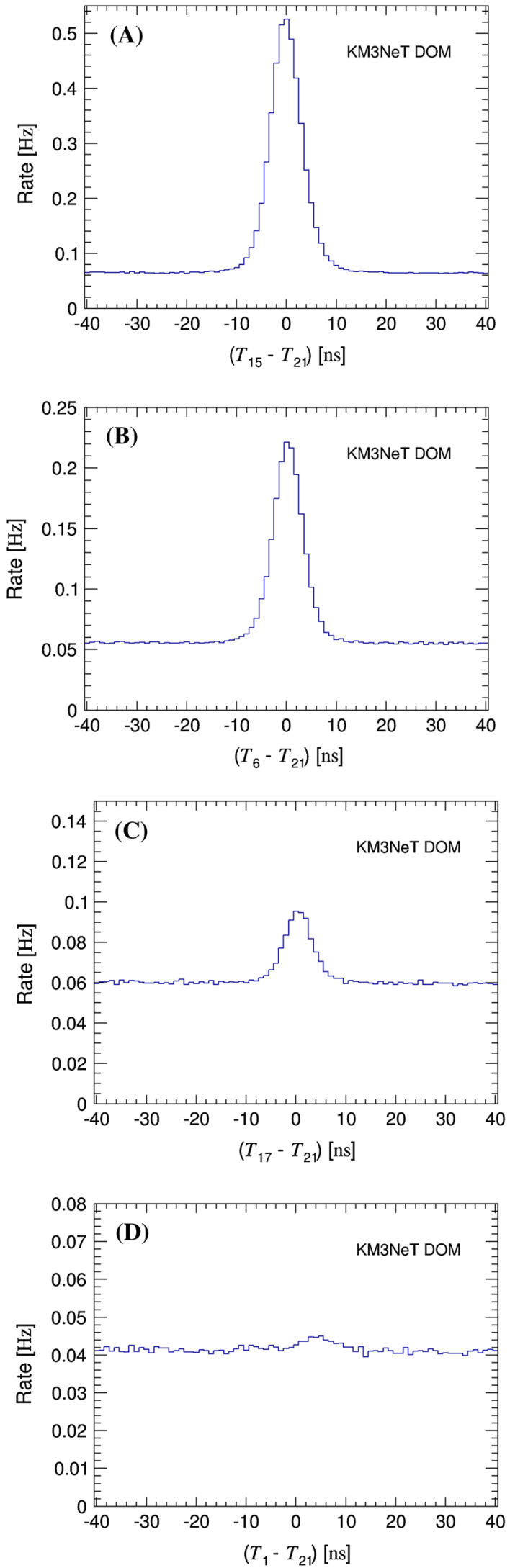

Fig. 4 Distribution of time differences between two PMTs of the DOM, with an angular separation of $\mathbf{a} 33^{\circ}, \mathbf{b} 65^{\circ}, \mathbf{c ~} 120^{\circ}$ and $\mathbf{d} 165^{\circ}$

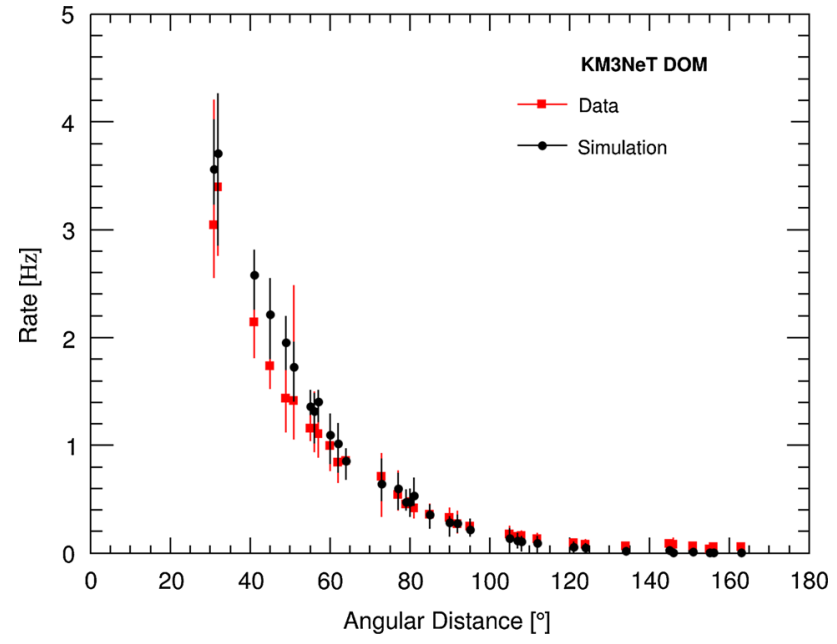

Fig. 5 The rate as a function of angular separation of the fitted coincidence signal is shown as a function of separation for both data (red squares) and simulation (black dots)

PMT time by $100 \mathrm{~ns}$ multiplied by its internal PMT index (1-31), the instantaneous combinatorial background can be measured. In Fig. $6 \mathrm{~b}-\mathrm{d}$ the lower red curve shows the coincidence rates with this combinatorial background subtracted. These curves thus show the evolution of the genuine ${ }^{40} \mathrm{~K}$ coincidences. As expected, the coincidence rates are completely stable at 340,30 and $2.7 \mathrm{~Hz}$ for twofold, threefold and fourfold coincidences, respectively, which agrees perfectly with the ${ }^{40} \mathrm{~K}$ simulations. At a coincidence level of four little contribution from bioluminescence is observed.

\section{Analysis}

The photon counting capability and the directionality provided by the photocathode segmentation will enable a single DOM to identify muons and to be sensitive to their arrival directions, as demonstrated in Fig. 7.

In Fig. 7a, the event rate is shown as a function of the coincidence level. The coincidence level is defined as the number of PMTs having a detected hit within a $20 \mathrm{~ns}$ time window. Below a coincidence level of six, the measured event rate is in good agreement with the event rate given by the simulation of the ${ }^{40} \mathrm{~K}$ decays [21]. The singles rate (coincidence level 1 ) is sensitive to the attenuation length in water and also has a contribution from bioluminescence, that is not simulated. This extra singles rate also gives an additional combinatorial contribution to the twofold coincidences. At the higher coincidence levels the simulation is in good agreement with the data. The rates decrease rapidly as a function of the coincidence level as every level increase leads to an extra factor in the acceptance of AРMT $/ 4 \pi d^{2}$, where $\mathrm{d}$ is the distance form the ${ }^{40} \mathrm{~K}$ decay to the PMT and $\mathrm{A}_{\mathrm{PMT}}$ is the sensitive area of 

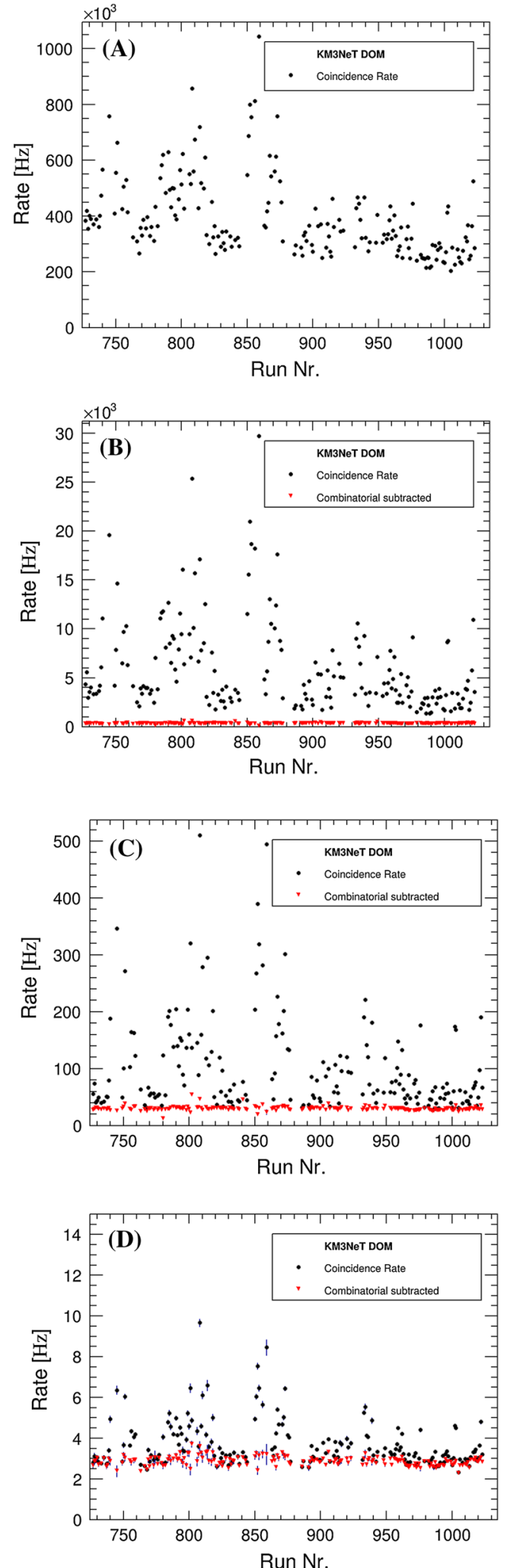

Fig. 6 Aggregate rates as a function of run number for a singles, $\mathbf{b}$ twofold coincidence, $\mathbf{c}$ threefold coincidence and $\mathbf{d}$ fourfold coincidence rates. The lower red points show the rates with combinatorial background subtracted and gives the true coincidences from ${ }^{40} \mathrm{~K}$. The runs cover the period from July to December 2013
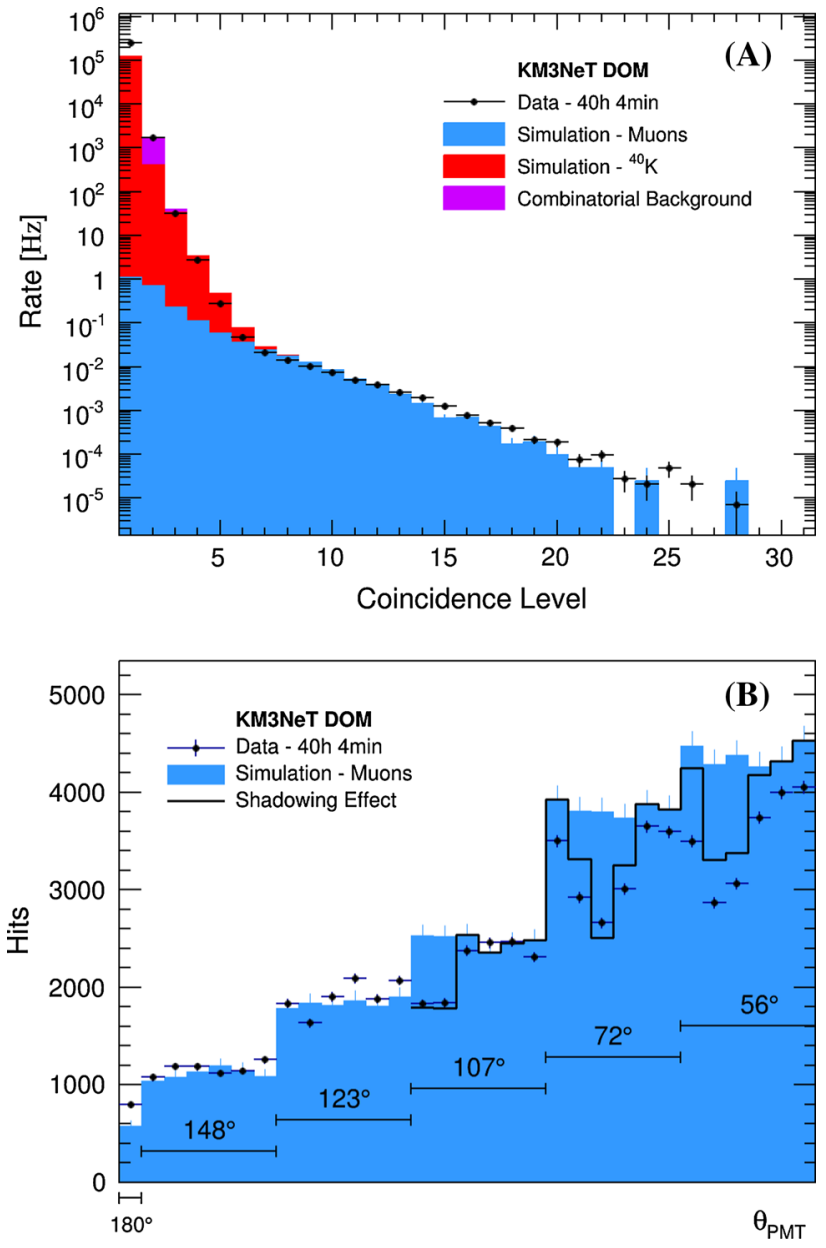

Fig. 7 a The rate of events as a function of the coincidence level (number of PMTs with signal in a $20 \mathrm{~ns}$ time window). Black dots correspond to data while coloured histograms represent simulations (muons in blue,

${ }^{40} \mathrm{~K}$ in red and accidental coincidences in purple). $\mathbf{b}$ The number of hits as a function of the zenith position of the centre of the PMT for coincidence levels above seven. One PMT is looking downward $\left(180^{\circ}\right)$. The others are grouped by six at five different angles. The black dots are data, the blue histogram is simulation of atmospheric muons and the black histogram show the calculated effect of the shadowing by the ANTARES electronics cylinder

the PMT. This leads to a rapidly decreasing volume of water inside which the DOM is sensitive to the ${ }^{40} \mathrm{~K}$ decays and therefore a rapid decrease in rate. Above the coincidence level of seven, the signals from atmospheric muons dominate. The simulation of the atmospheric muons was performed using a parameterisation of the measured muon flux and multiplicity for the $2375 \mathrm{~m}$ depth of the DOM [22]. An excellent agreement is seen between data and simulation of atmospheric muons. Therefore, with a single DOM muons are unambiguously identified using coincidences of only eight PMTs.

In Fig. 7b, the number of hits detected by each PMT is shown as a function of their position in terms of zenith angle, corresponding to the rings of PMTs in the DOM. For this 
figure a cut was applied at a coincidence level larger than seven, which selects a pure muon sample. With the decrease in zenith angle of the PMT, the rate increases, since atmospheric muons come from above. There is good agreement between data and the atmospheric muon simulation. The drop in hit counts for PMTs in the upper three rings is due to a shadowing effect of the electronics cylinder of the ANTARES line. Since the final KM3NeT suspension of the DOM will not cause such shadowing, incorporating the effect in the Monte Carlo was not considered. Instead the loss in efficiency for the PMTs was calculated assuming all muons arrive exactly vertically and are uniformly distributed horizontally. The black histogram in Fig. $7 \mathrm{~b}$ shows that this first order estimate reproduces the effect quite reasonably.

\section{Conclusion}

The novel digital optical module designed with large photocathode area segmented by the use of 31 small PMTs has been tested in deep sea. The DOM was connected to the instrumentation line of the ANTARES telescope at a depth of around $2375 \mathrm{~m}$. Data taking was possible continuously from the moment it was connected and is still on-going. The baseline counting rates are stable at around $8 \mathrm{kHz}$ per PMT with rates averaged over 10 minutes observed as high as $1.2 \mathrm{MHz}$ for the full DOM. The directional capabilities of the DOM have allowed for the ANTARES support structure to be identified as a cause of excitation of bioluminescent activity. The signals from ${ }^{40} \mathrm{~K}$ decay have been used to show the good timing and directional capabilities of the DOM. These signals will provide a straightforward intra-DOM timing calibration in KM3NeT. With the single DOM it was possible at the level of eightfold coincidences to select a virtually background free sample of atmospheric muons, demonstrating the background suppression capabilities of the new design. Finally using the pure muon sample it was possible to demonstrate the sensitivity of the DOM to the arrival direction of the muons. This design provides a highly capable optical module for future neutrino telescopes.

\footnotetext{
Acknowledgments The authors wish to thank the ANTARES collaboration for agreeing to the installation of the DOM in the detector and for the technical support given. The research leading to these results has received funding from the European Community Sixth Framework Programme under Contract 011937 and the Seventh Framework Programme under Grant Agreement 212525.
}

Open Access This article is distributed under the terms of the Creative Commons Attribution License which permits any use, distribution, and reproduction in any medium, provided the original author(s) and the source are credited.

Funded by $\mathrm{SCOAP}^{3}$ / License Version CC BY 4.0.

\section{References}

1. KM3NeT Technical Design Report. ISBN 978-90-6488-033-9. http://km3net.org/TDR/TDRKM3NeT

2. M.G. Aartsen et al. (IceCube Collaboration), First observation of PeV-energy neutrinos with IceCube. Phys. Rev. Lett. 111, 021103 (2013)

3. M.G. Aartsen et al. (IceCube Collaboration), Evidence for highenergy extraterrestrial neutrinos at the IceCube detector. Science 342, 947 (2013). doi:10.1126/science. 1242856

4. F. Halzen, D. Hooper, High-energy neutrino astronomy: the cosmic ray connection. Rep. Prog. Phys. 65, 1025 (2002)

5. W. Bednarek, TeV neutrinos from microquasars in compact massive binaries. Astrophys. J. 631, 466 (2005)

6. F.W. Stecker, Are diffuse high energy neutrinos and $\gamma$-rays from starburst galaxies observable? Astropart. Phys. 26, 398 (2007)

7. F.W. Stecker, A note on high energy neutrinos from AGN cores. Phys. Rev. D 72, 107301 (2005)

8. A. Kappes et al., Potential neutrino signals from Galactic $\gamma$-ray sources. Astrophys. J. 656, 870 (2007)

9. S. Adrián-Martínez et al. (ANTARES Collaboration), Search for cosmic neutrino point sources with four years of data from the ANTARES telescope. Astrophys. J. 760, 53 (2012)

10. M. Ageron et al. (ANTARES Collaboration), ANTARES: the first undersea neutrino telescope. Nucl. Instrum. Methods A 656, 11 (2011)

11. S. Adrián-Martínez et al. (KM3NeT Collaboration), Expansion cone for the 3-inch PMTs of the KM3NeT optical modules. JINST 8, T03006 (2013)

12. P. Timmer et al., Very low power, high voltage base for a Photo Multiplier Tube for the KM3NeT deep sea neutrino telescope. JINST 5, C12049 (2010)

13. A. Zwart et al. (On behalf of the KM3NeT collaboration), A TDC for the characterization of KM3NeT PMTs. Nucl. Instrum. Methods A 725, 170 (2013)

14. S. Anvar et al., Design and implementation of a nanosecond time-stamping readout system-on-chip for photo-detectors. Nucl. Instrum. Methods A 735, 587 (2014)

15. J. Hogenbirk et al., A photonic readout and data acquisition system for deep-sea neutrino telescopes. Nucl. Instrum. Methods A 626627, S166 (2011)

16. A. Belias, K. Manolopoulos, Readout and data acquisition for KM3NeT. AIP Conf. Proc. 1535, 260 (2013)

17. P. Amram et al. (ANTARES collaboration), The ANTARES optical module. Nucl. Instrum. Methods A 484, 369 (2002)

18. E.G. Anassontzis et al., Nucl. Instrum. Methods A 479, 439 (2002)

19. S. Aiello et al., The optical modules of the phase- 2 of the NEMO project. J. Instrum. 8, P07001 (2013)

20. J.A. Aguilar et al. (ANTARES Collaboration), Time calibration of the ANTARES neutrino telescope. Astropart. Phys. 34, 539 (2011)

21. B. Herold (KM3NeT Collaboration), Study of 40K-induced rates for a KM3NeT design option with multi-PMT optical modules. Nucl. Instrum. Methods A 626-627, S234 (2011)

22. Y. Becherini et al., A parameterisation of single and multiple muons in the deep water or ice. Astropart. Phys. 25, 1 (2006) 